\title{
Growth, fabrication, and testing of bismuth tri-iodide semiconductor radiation detectors
}

\author{
Sasmit S. Gokhale ${ }^{a, *}$, Hyuksu $\operatorname{Han}^{b}$, James E. Baciak ${ }^{a}$, Kelly A. Jordan ${ }^{a}$ and \\ Juan C. Nino ${ }^{b}$ \\ ${ }^{a}$ Nuclear Engineering program at the Department of Materials Science and Engineering, \\ 100 Rhines Hall, \\ University of Florida, \\ Gainesville, FL 32611 USA \\ ${ }^{b}$ Department of Materials Science and Engineering, \\ 100 Rhines Hall, \\ University of Florida, \\ Gainesville, FL 32611 USA \\ E-mail: sasmitg@ufl.edu \\ Tel: +1-352-871-9034
}

ABSTRACT: Bismuth tri-iodide $\left(\mathrm{BiI}_{3}\right)$ is an attractive material for high energy resolution radiation detectors. For the purpose of this research, detectors were fabricated using single crystals grown from ultra-pure $\mathrm{BiI}_{3}$ powder; synthesized by the Physical Vapor Transport (PVT) technique. This technique yielded powder with total impurity level of $7.9 \mathrm{ppm}$. Efforts were also made to purify commercial $\mathrm{BiI}_{3}$ powder using a custom-built Traveling Zone Refining (TZR) system. Initial trial runs were successful in reducing the total impurity level of the commercial powder from $200 \mathrm{ppm}$ to less than $50 \mathrm{ppm}$. Using the modified vertical Bridgman technique and a customized sharp tip ampoule, a large $\mathrm{BiI}_{3}$ single crystal was grown. The crystal had a surface area of $2.2 \mathrm{~cm}^{2}$ and a thickness of $0.8 \mathrm{~cm}$, which corresponds to a volume of $1.78 \mathrm{~cm}^{3}$. Radiation detectors were fabricated and then tested by measuring their electrical characteristics and radiation response. An alpha particle spectrum (using a ${ }^{241} \mathrm{Am} \alpha$-source) was recorded at room temperature with a $\mathrm{BiI}_{3}$ detector $0.09 \mathrm{~cm}$ thick and with a surface area of 0.16 $\mathrm{cm}^{2}$. The electron mobility was estimated to be $433 \pm 79 \mathrm{~cm}^{2} / \mathrm{Vs}$.

KEYWORDS: Room temperature semiconductor detectors; Semiconductor materials; Radiation spectrometers.

\footnotetext{
${ }^{*}$ Corresponding author.
} 


\section{Introduction}

Gamma-ray spectroscopy is of interest for a number of applications including, astronomy, medical imaging, reactor engineering, and nuclear security. These applications require detectors with high efficiency and excellent energy resolution. Detectors fabricated from wide band-gap semiconductor materials possess the properties necessary for high detection efficiency and energy resolution; in addition, they can also operate at room temperature without the need for cryogenic cooling [1]. Over the years a number of compound semiconductors such as cadmium zinc telluride (CdZnTe) [2], cadmium telluride (CdTe) [3], and thallium bromide ( $\mathrm{TlBr}$ ) [4] have been investigated and successfully applied for room temperature radiation detection.

Iodine based semiconductors such as mercuric iodide $\left(\mathrm{HgI}_{2}\right)$ and lead iodide $\left(\mathrm{PbI}_{2}\right)$ possess all of the necessary properties required for room temperature gamma-ray spectroscopy and they have been successfully used as radiation detectors. There are however some material and growth issues with both $\mathrm{HgI}_{2}$ and $\mathrm{PbI}_{2}$ [5] and as a result these materials have not gained much popularity. $\mathrm{BiI}_{3}$ is a semiconductor material, which has properties similar to $\mathrm{HgI}_{2}$ and $\mathrm{PbI}_{2}$, but it does not share their major limitations [6]. $\mathrm{BiI}_{3}$ has high effective atomic number (since $\mathrm{Z}_{\mathrm{Bi}}=$ 83 and $\left.\mathrm{Z}_{\mathrm{I}}=53\right)$ and high density $\left(5.78 \mathrm{~g} / \mathrm{cm}^{3}\right)$ and thus has a very good photon stopping power [7]. The large band-gap energy $(1.67 \mathrm{eV}[8])$ of $\mathrm{BiI}_{3}$ will allow it to function as a room temperature radiation detector without any cooling mechanism. $\mathrm{HgI}_{2}, \mathrm{PbI}_{2}$, and $\mathrm{BiI}_{3}$ all have a layered crystal structure with weak van der Waals bonding between the layers, and therefore the crystals are mechanically soft and cleave easily [9]. Unlike $\mathrm{HgI}_{2}, \mathrm{BiI}_{3}$ does not undergo phase transition since it has only one phase (rhombohedral [10]) which simplifies its crystal growth; furthermore, it is more robust and less toxic than $\mathrm{PbI}_{2}$ [11], [1]. MCNP calculations have also predicted that $\mathrm{BiI}_{3}$ has a much higher (by a factor of 2-3) photopeak efficiency than CdZnTe [5]. 
All of these properties have made $\mathrm{BiI}_{3}$ an attractive option for further development as a room temperature radiation detector.

\section{2. $\mathrm{BiI}_{3}$ ultra-pure powder synthesis}

The ultra-pure $(6 \mathrm{~N})$ powder used for growing $\mathrm{BiI}_{3}$ single crystals was synthesized by the physical vapor transport (PVT) technique. Commercially available lumps of ultra-pure metal bases $\mathrm{Bi}$ and $\mathrm{I}_{2}$ were used for the powder synthesis. The reaction between bismuth and iodine vapors is facilitated by the flow of Argon (Ar) gas through a quartz tube in a two zone tube furnace. A boat with a lump of iodine is placed near one end of the quartz tube in a heating zone at $150{ }^{\circ} \mathrm{C}$ to avoid $\mathrm{BiI}_{3}$ deposition on the quartz boat. The boat with a lump of bismuth is placed near the center of the quartz tube in a zone heated to $400{ }^{\circ} \mathrm{C}$. The synthesized $\mathrm{BiI}_{3}$ powder is collected on a Teflon ${ }^{\circledR}$ sheet at the other end of the tube, which is maintained at a temperature close to room temperature. The reaction apparatus and the tube furnace can be seen in figure 1 .

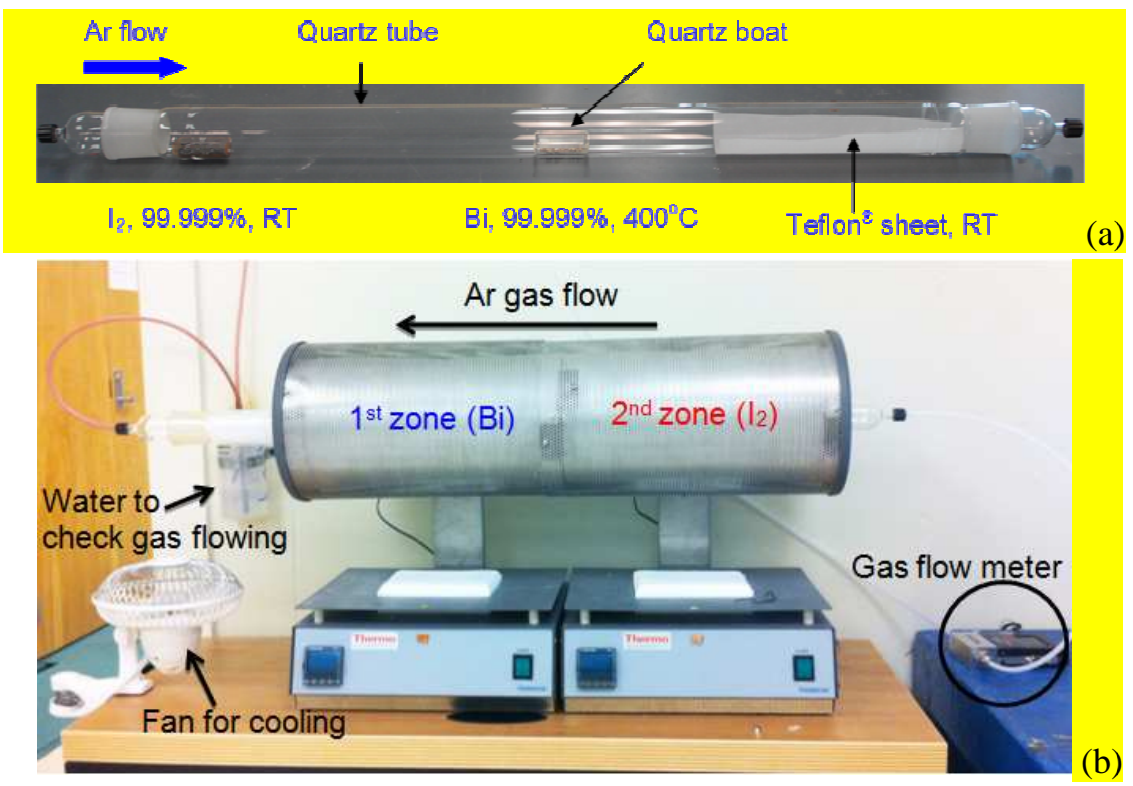

Figure 1. Reaction apparatus for $\mathrm{BiI}_{3}$ powder synthesis. (a) Quartz tube, (b) two zone furnace setup.

The impurity analysis was carried out by ICP-AES (inductively coupled plasma - atomic emission spectroscopy). $\mathrm{BiI}_{3}$ powder was dissolved in $10 \%$ trace metal grade $\mathrm{HCl}$ solution with 
a concentration of $10 \mathrm{mg} / \mathrm{ml}$. The results of the analysis are summarized in table 1 . Total impurity level of the PVT synthesized powder was measured to be approximately $7.9 \mathrm{ppm}$. Some of the elements probed had concentrations below the detection limit of the ICP-AES, and thus were noted as ND (Not Detected) in the table. The total impurity level indicates the concentration of the elements that can be probed by ICP-AES only and not all possible impurities.

Table 1. Impurity analysis of PVT synthesized $\mathrm{BiI}_{3}$ powder.

\begin{tabular}{cccccccc}
\hline Element & $\begin{array}{c}\mathrm{C} . \\
(\mathrm{ppm})\end{array}$ & Element & $\begin{array}{c}\mathrm{C} . \\
(\mathrm{ppm})\end{array}$ & Element & $\begin{array}{c}\mathrm{C} . \\
(\mathrm{ppm})\end{array}$ & Element & $\begin{array}{c}\mathrm{C} . \\
(\mathrm{ppm})\end{array}$ \\
\hline \hline $\mathrm{Cu}$ & 0.3 & $\mathrm{Li}$ & $\mathrm{ND}$ & $\mathrm{Cr}$ & $\mathrm{ND}$ & $\mathrm{Mo}$ & 0.1 \\
$\mathrm{Ag}$ & $\mathrm{ND}$ & $\mathrm{Be}$ & $\mathrm{ND}$ & $\mathrm{Mn}$ & $\mathrm{ND}$ & $\mathrm{Cd}$ & 0.1 \\
$\mathrm{~Pb}$ & $\mathrm{ND}$ & $\mathrm{Mg}$ & 1.1 & $\mathrm{Co}$ & $\mathrm{ND}$ & $\mathrm{Sb}$ & $\mathrm{ND}$ \\
$\mathrm{Ni}$ & $\mathrm{ND}$ & $\mathrm{Ca}$ & $\mathrm{ND}$ & $\mathrm{Zn}$ & $\mathrm{ND}$ & $\mathrm{Tl}$ & $\mathrm{ND}$ \\
$\mathrm{Fe}$ & $\mathrm{ND}$ & $\mathrm{K}$ & $\mathrm{ND}$ & $\mathrm{As}$ & 1.3 & $\mathrm{~B}$ & 4.3 \\
$\mathrm{Na}$ & $\mathrm{ND}$ & $\mathrm{Ti}$ & $\mathrm{ND}$ & $\mathrm{Se}$ & $\mathrm{ND}$ & $\mathrm{Al}$ & $\mathrm{ND}$ \\
$\mathrm{Si}$ & $\mathrm{ND}$ & $\mathrm{V}$ & $\mathrm{ND}$ & $\mathrm{Sr}$ & $\mathrm{ND}$ & $\mathrm{Ba}$ & 0.7 \\
\hline
\end{tabular}

The impurity level of the commercially acquired $\mathrm{BiI}_{3}$ powder manufactured by $\mathrm{MV}$ Laboratories (nominal purity $5 \mathrm{~N}$ ) was also measured in order to compare it to that of the PVT synthesized powder. The results of the ICP-AES analysis can be seen in table 2. As can be seen from the table, the total impurity level of the commercial powder was around $116.92 \mathrm{ppm}$. High concentrations of metallic impurities, such as $\mathrm{Ni}(63.73 \mathrm{ppm})$ and $\mathrm{Fe}(12.83 \mathrm{ppm})$, were also detected in the commercial powder.

Table 2. Impurity analysis of commercially synthesized $\mathrm{BiI}_{3}$ powder.

\begin{tabular}{cccccccc}
\hline Element & $\begin{array}{c}\mathrm{C} . \\
(\mathrm{ppm})\end{array}$ & Element & $\begin{array}{c}\mathrm{C} . \\
(\mathrm{ppm})\end{array}$ & Element & $\begin{array}{c}\mathrm{C} . \\
(\mathrm{ppm})\end{array}$ & Element & $\begin{array}{c}\mathrm{C} . \\
(\mathrm{ppm})\end{array}$ \\
\hline \hline $\mathrm{Cu}$ & $\mathrm{ND}$ & $\mathrm{Li}$ & $\mathrm{ND}$ & $\mathrm{Cr}$ & $\mathrm{ND}$ & $\mathrm{Mo}$ & 0.75 \\
$\mathrm{Ag}$ & 4.47 & $\mathrm{Be}$ & $\mathrm{ND}$ & $\mathrm{Mn}$ & $\mathrm{ND}$ & $\mathrm{Cd}$ & 0.1 \\
$\mathrm{~Pb}$ & $\mathrm{ND}$ & $\mathrm{Mg}$ & 0.58 & $\mathrm{Co}$ & 1.57 & $\mathrm{Sb}$ & $\mathrm{ND}$ \\
$\mathrm{Ni}$ & 63.73 & $\mathrm{Ca}$ & 6.59 & $\mathrm{Zn}$ & $\mathrm{ND}$ & $\mathrm{Tl}$ & $\mathrm{ND}$ \\
$\mathrm{Fe}$ & 12.83 & $\mathrm{~K}$ & 22.18 & $\mathrm{As}$ & $\mathrm{ND}$ & $\mathrm{B}$ & 2.36 \\
$\mathrm{Na}$ & $\mathrm{ND}$ & $\mathrm{Ti}$ & 0.73 & $\mathrm{Se}$ & $\mathrm{ND}$ & $\mathrm{Al}$ & 1.03 \\
$\mathrm{Si}$ & $\mathrm{ND}$ & $\mathrm{V}$ & $\mathrm{ND}$ & $\mathrm{Sr}$ & $\mathrm{ND}$ & $\mathrm{Ba}$ & $\mathrm{ND}$ \\
\hline
\end{tabular}

Efforts were also made to purify commercial $\mathrm{BiI}_{3}$ powder using a custom built traveling zone refining (TZR) system. In order to purify the $\mathrm{BiI}_{3}$ powder, it was sealed in an ampoule, 
which was then mounted into the TZR setup. The TZR setup consists of a band heater, which establishes a narrow molten zone, which is then moved along the length of the ampoule. The moving molten zone causes the impurities to be segregated at one end of the ampoule, which is then separated from the purified bulk. Initial trial runs were successful in reducing the total impurity level of the commercial powder from 200 ppm to less than 50 ppm. Figure 2 shows the TZR system.

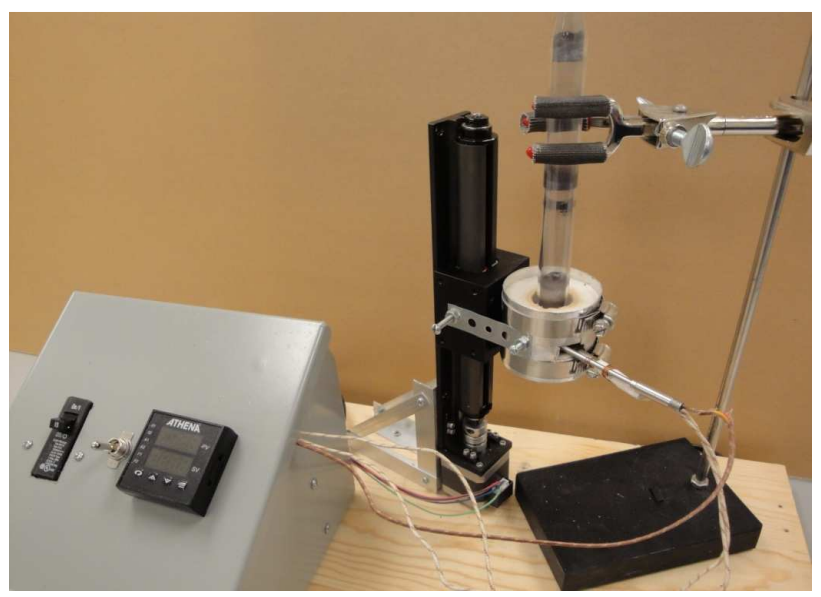

Figure 2. Traveling zone refining system with vertical ampoule and heating collar with controlled vertical displacement.

The powder was sealed in a Pyrex glass ampoule under a vacuum of $10^{-7}$ Torr. The molten zone width was approximately 1 , while the $\mathrm{BiI}_{3}$ powder in the ampoule would typically occupy a length of about $8 \mathrm{~cm}$ which results in a molten zone width to total charge length ratio of about 1:8. Zone refining was then conducted for $90-100$ passes at a molten zone travel speed of 4.4 $\mathrm{cm} / \mathrm{hr}$. These refining parameters are comparable to those used in previous works [12], [13], and [14]. The Impurity analysis was carried out by ICP-AES and the result is summarized in table 3. Similar decrease in impurity concentration was observed in previous work [14]. The total impurity level of $26.3 \mathrm{ppm}$ was achieved by the zone refining process. This impurity level is not as low as that of the PVT synthesized high pure $\mathrm{BiI}_{3}$ powder, however as can be seen from table 3 , the element with the highest concentration in the TZR purified powder is boron. The most 
likely source of boron is the Pyrex glass ampoule in which the powder was sealed for purification and therefore this contamination can be avoided by using quartz ampoules.

Table 3. Impurity analysis of TZR purified $\mathrm{BiI}_{3}$ powder.

\begin{tabular}{cccccccc}
\hline Element & $\begin{array}{c}\mathrm{C} . \\
(\mathrm{ppm})\end{array}$ & Element & $\begin{array}{c}\mathrm{C} . \\
(\mathrm{ppm})\end{array}$ & Element & $\begin{array}{c}\mathrm{C} . \\
(\mathrm{ppm})\end{array}$ & Element & $\begin{array}{c}\mathrm{C} . \\
(\mathrm{ppm})\end{array}$ \\
\hline \hline $\mathrm{Cu}$ & $\mathrm{ND}$ & $\mathrm{Li}$ & $\mathrm{ND}$ & $\mathrm{Cr}$ & $\mathrm{ND}$ & $\mathrm{Mo}$ & $\mathrm{ND}$ \\
$\mathrm{Ag}$ & 4.2 & $\mathrm{Be}$ & $\mathrm{ND}$ & $\mathrm{Mn}$ & $\mathrm{ND}$ & $\mathrm{Cd}$ & 0.1 \\
$\mathrm{~Pb}$ & $\mathrm{ND}$ & $\mathrm{Mg}$ & $\mathrm{ND}$ & $\mathrm{Co}$ & 0.1 & $\mathrm{Sb}$ & $\mathrm{ND}$ \\
$\mathrm{Ni}$ & 1.6 & $\mathrm{Ca}$ & $\mathrm{ND}$ & $\mathrm{Zn}$ & $\mathrm{ND}$ & $\mathrm{Tl}$ & $\mathrm{ND}$ \\
$\mathrm{Fe}$ & $\mathrm{ND}$ & $\mathrm{K}$ & $\mathrm{ND}$ & $\mathrm{As}$ & 2.7 & $\mathrm{~B}$ & 17.5 \\
$\mathrm{Na}$ & $\mathrm{ND}$ & $\mathrm{Ti}$ & $\mathrm{ND}$ & $\mathrm{Se}$ & $\mathrm{ND}$ & $\mathrm{Al}$ & $\mathrm{ND}$ \\
$\mathrm{Si}$ & $\mathrm{ND}$ & $\mathrm{V}$ & $\mathrm{ND}$ & $\mathrm{Sr}$ & $\mathrm{ND}$ & $\mathrm{Ba}$ & 0.1 \\
\hline
\end{tabular}

In general, the TZR method has some advantages over PVT, viz. it has a much higher yield and lower cost compared to the PVT method. The TZR setup can purify approximately 100 grams of powder in a week with some losses to the impure tails, while only 1 gram of $\mathrm{BiI}_{3}$ powder can be synthesized in three days by the PVT method using the setup described above. Some $I_{2}$ deposition was seen on the ampoule wall after zone refining, therefore it was assumed that the stoichiometry was not perfect. It has been reported in previously that zone refining $\mathrm{BiI}_{3}$ causes some losses of iodine. However, the stoichiometry of the powder can be restored by sublimation in the presence of free iodine before crystal growth [14] [15]. The decomposition of the powder during zone refining can be minimized by backfilling the ampoule with an inert gas such as argon. The main drawback of the TZR setup is that further reduction in the impurities would require an exponential increase in the number of passes and hence the total time required. The synthesis of powder by the PVT method can be scaled up by running multiple furnaces simultaneously, but considering the costs for the additional furnaces, the raw metallic bismuth and iodine sources, and the Ar gas, it is worthwhile to use the TZR method for purifying $\mathrm{BiI}_{3}$ powder for future research. 


\section{Crystal growth and detector fabrication}

The $\mathrm{BiI}_{3}$ crystal growth by the modified vertical Bridgman method was carried out using the same procedure as described elsewhere [16]. For crystal growth the PVT synthesized ultra-pure powder was sealed in a Pyrex glass ampoule under a vacuum of $10^{-7}$ Torr. The customized sharp tip ampoule (figure 3(a)), with a tip length of $5 \mathrm{~cm}$ and end angle of $70^{\circ}$, was purchased from Southern Scientific, INC. The crystal growth resulted in a polycrystalline boule. The tip region of the boule had multiple grains, while the main bulk of the boule was a single crystal. A large $\mathrm{BiI}_{3}$ single crystal with a surface area of $2.2 \mathrm{~cm}^{2}$ and a thickness of $0.8 \mathrm{~cm}$, which corresponds to a volume of $1.7 \mathrm{~cm}^{3}$ (figure 3(b)) was extracted from the bulk.

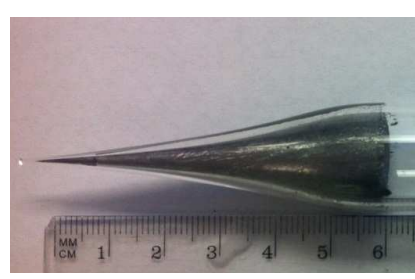

(a)

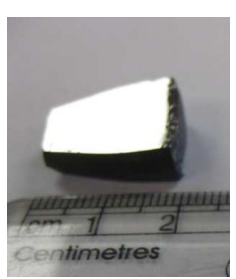

(b)

Figure 3. (a) Customized Sharp tip ampoule and (b) the $\mathrm{BiI}_{3}$ large single crystal extracted (surface area: $2.2 \mathrm{~cm}^{2}$, thickness: $0.8 \mathrm{~cm}$ ).

The single crystal was cleaved along the direction perpendicular to the [001] plane by using a razor blade, and then the (001) surfaces of the cleaved $\mathrm{BiI}_{3}$ single crystal were prepared by peeling off the top layer using adhesive tape to obtain a mirror surface. The mirror surfaces were sputter coated with Au metal using a Kurt J. Lesker ${ }^{\circledR}$ CMS-18 multi target sputter system. The thickness of the sputtered electrode was around $100 \mathrm{~nm}$. The sputtering was conducted in a vacuum chamber with a pressure of $10^{-8}$ Torr, at a deposition rate of $0.5 \mathrm{~nm} / \mathrm{s}$ and at room temperature. It has been recently shown that Au provides the best electrode compatibility with $\mathrm{BiI}_{3}[17]$. Both Au and Pd have been previously used as contacts for $\mathrm{BiI}_{3}$ [18] single crystals and polycrystalline films [19].

The following results focus mainly on the detectors fabricated from crystals grown using PVT synthesized $\mathrm{BiI}_{3}$ powder. 


\section{Detector characterization}

\subsection{Crystal characterization}

The dislocation density of the as grown single crystals has been examined previously by the etch pit density (EPD) method and was determined to be on the order of $10^{4} / \mathrm{cm}^{2}$ [20].

\subsection{Electrical characterization}

The detectors were characterized by measuring their electrical properties and radiation response. $\mathrm{I}-\mathrm{V}$ measurements were carried out to determine the behavior of the contacts (ohmic or Schottky) and to determine the resistivity.

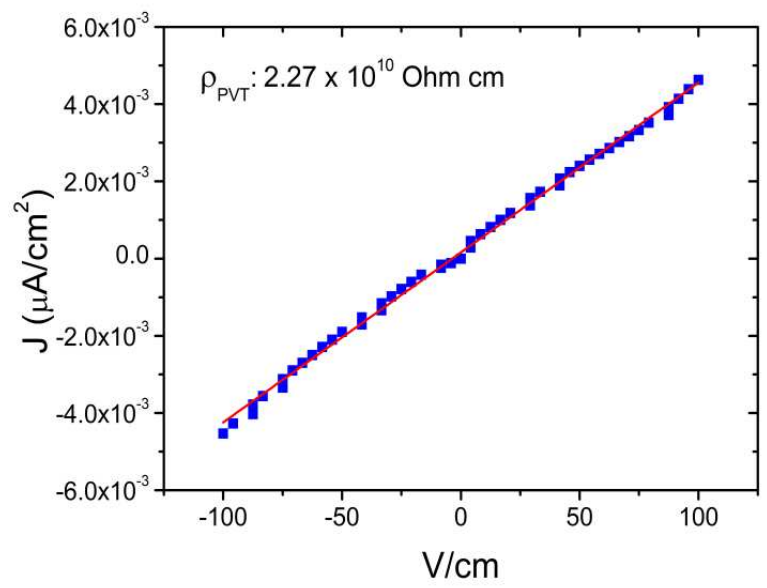

Figure 4. I-V characterization of the PVT single crystals. $\boldsymbol{\rho}_{\mathrm{PVT}}$ is the resistivity of the $\mathrm{PVT} \mathrm{BiI}_{3}$ single crystal.

It was observed that the gold electrodes sputtered onto the detectors exhibited ohmic behavior. As can be seen from figure 4, the resistivity value obtained from the I-V curves was on the order of $10^{10} \Omega$-cm. This value is orders of magnitude higher than the previously reported value of resistivity of the $\mathrm{BiI}_{3}$ detectors which were fabricated from crystals grown using commercial powder [16]. Thus it may be hypothesized that the increase in resistivity may be correlated to the increase in the purity of the crystals. Resistivity values in the range of $10^{12}$ $10^{13} \Omega$-cm were measured for $\mathrm{BiI}_{3}$ platelets grown by the physical vapor deposition (PVD) [19], [21], and [22]. Resistivity values in the range of $10^{10} \Omega$-cm were reported for $\mathrm{BiI}_{3}$ platelets grown by PVD as well as for single crystals grown by the Bridgman method [6], [7], and [23]. 
Growth of $\mathrm{BiI}_{3}$ from zone refined powder has also been reported with a resistivity in the range of $10^{6} \Omega-\mathrm{cm}[24]$.

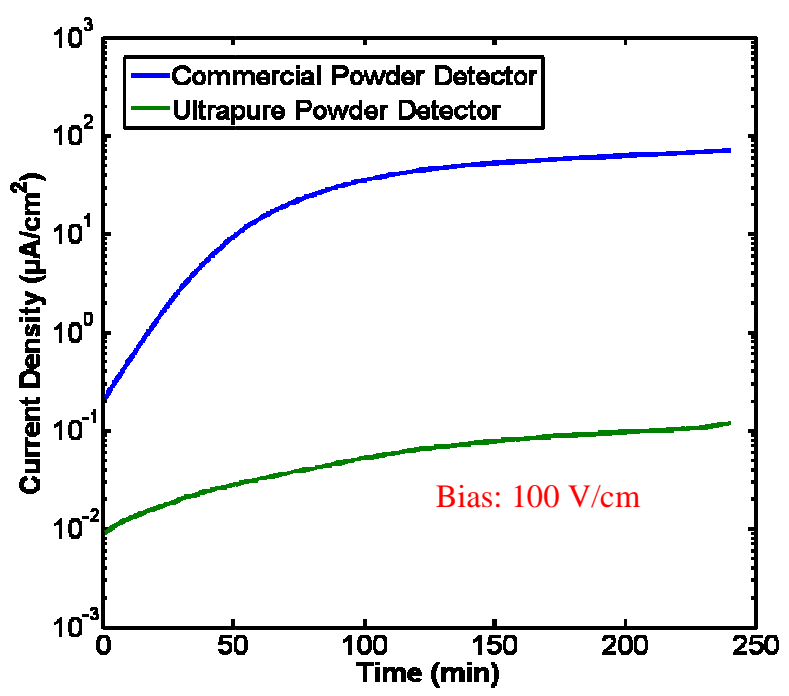

Figure 5. Comparison of leakage current between $\mathrm{BiI}_{3}$ detectors made with crystals grown from commercially acquired powder and PVT synthesized powder.

Leakage current measurement was performed to determine the variation of leakage current flowing through the device to understand the behavior of the detector under a constant bias for prolonged times. The measurement was carried out using a Keithley 6517B electrometer, which supplies voltage and measures the current through the detector as a function of time. Figure 5 shows a comparison between the leakage currents measured for two prototype detectors, one fabricated from commercial powder crystal and the other from ultrapure powder crystal. Both the detectors were subjected to an electric field of $100 \mathrm{~V} / \mathrm{cm}$. As can be seen from the figure, the leakage current in the ultrapure $\mathrm{BiI}_{3}$ detectors is orders of magnitude lower than that in the commercial $\mathrm{BiI}_{3}$ detectors. Furthermore, the ultrapure detector performed consistently during subsequent tests and had low leakage current throughout. This decrease in the leakage current density of the PVT synthesized $\mathrm{BiI}_{3}$ detectors is due to their higher resistivity which in turn may be attributed to the decrease in the impurity concentration. 


\subsection{Room temperature radiation response}

Radiation responses of the detectors made with PVT synthesized $\mathrm{BiI}_{3}$ were investigated using Amptek A250 charge sensitive pre-amplifiers and standard NIM electronics. Spectrum acquisition was carried out using an ORTEC $^{\circledR}$ EASY-MCA and Maestro 32 spectrum acquisition software. A prototype detector of thickness $0.092 \mathrm{~cm}$ and with a surface area of 0.16 $\mathrm{cm}^{2}$ was fabricated to measure the radiation response. An alpha source was used for characterizing the detector radiation response.

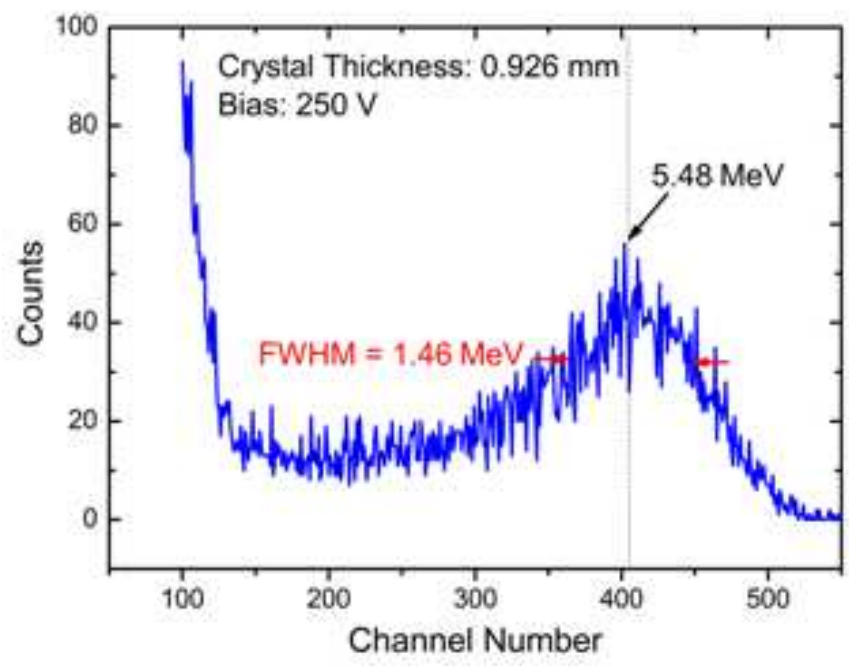

Figure 6. ${ }^{241} \mathrm{Am} \boldsymbol{\alpha}$-particle spectrum obtained with a $\mathrm{BiI}_{3}$ detector made with a crystal grown from PVT synthesized powder at room temperature.

Figure 6 shows the $\alpha$-particle spectrum recorded, by irradiating the cathode of a $\mathrm{BiI}_{3}$ detector at room temperature. The $\alpha$-particle source used was an Americium-241 $\left({ }^{241} \mathrm{Am}\right)$ source from a smoke detector. The source activity was approximately $0.9 \mu \mathrm{Ci}$. A bias of $250 \mathrm{~V}$, corresponding to an electric field of $2600 \mathrm{~V} / \mathrm{cm}$, was applied to the detector and a spectrum was acquired with an amplifier shaping time of $2 \mu \mathrm{s}$. The full width at half maximum (FWHM) of the $5.48 \mathrm{MeV}$ peak was calculated to be $1.46 \mathrm{MeV}$. This value is better than the previously reported energy resolution for $\mathrm{BiI}_{3}[7]$. 


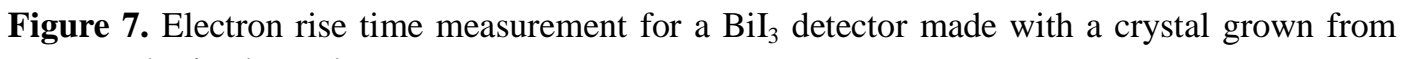
PVT synthesized powder.

The electron mobility was measured from the response of a detector to an ${ }^{241} \mathrm{Am}$ alpha source. The mobility was calculated by irradiating the cathode of the detector and measuring the rise times (figure 7) of the output pulses at the anode. The electron mobility was estimated to be $433 \pm 79 \mathrm{~cm}^{2} /$ Vs. The hole mobility could not be estimated because the signal generated at the cathode was too weak to measure. Previous research had reported the electron mobility to be 
$260 \pm 50 \mathrm{~cm}^{2} / \mathrm{Vs}$ for $\mathrm{BiI}_{3}$ single crystals grown by the vertical Bridgman method [16] and 4.4 $\mathrm{cm}^{2} / \mathrm{Vs}$ for $\mathrm{BiI}_{3}$ platelets grown by PVD [19]. Charge carrier mobility depends on the concentration of traps and defects in the detector crystal. Thus it may be hypothesized that the increased mobility that was measured may be due to the increased purity of the crystal, which in turn can improve crystal quality thereby resulting in a detector crystal with fewer traps and defects.

The poor hole transport indicate that single polarity charge sensing techniques [25] will be necessary for improving the detector performance. Poor hole transport can also explain the lack of detector response to gamma-radiation, since in detectors with planar electrodes the output signal generated by gamma-rays depends on both the electrons and holes due to the greater penetration depth of the gamma radiation.

\section{Conclusions}

Purification of the $\mathrm{BiI}_{3}$ powder to a high level $(6 \mathrm{~N})$ resulted in the overall improvement of properties of the $\mathrm{BiI}_{3}$ detectors. Ultrapure powder with an impurity level of less than $10 \mathrm{ppm}$ was synthesized by the physical vapor transport (PVT) technique. The resistivity of the single crystals grown from the PVT synthesized ultra-pure powder was found to be on the order of $10^{10} \Omega$-cm which is greater than the resistivity of crystals grown from commercial powder. Thus it may be hypothesized that the increase in resistivity may be correlated to the increase in the purity of the crystals. Commercial $\mathrm{BiI}_{3}$ with an initial impurity level of $200 \mathrm{ppm}$ was also purified by the traveling zone refining (TZR) technique in a custom made setup to an impurity level of $26.3 \mathrm{ppm}$. TZR offers a cheaper and less time consuming alternative to PVT. TZR purification may cause some loss of $\mathrm{I}_{2}$ and a change in stoichiometry. However, stoichiometry can be restored by sublimating the purified powder in the presence of free iodine prior to crystal growth. 
$\mathrm{BiI}_{3}$ single crystal of volume $1.78 \mathrm{~cm}^{3}$ was grown in a customized sharp tip ampoule using a modified vertical Bridgman furnace. The customized ampoule facilitates the consistent growth of boules from which large single crystals can be extracted..

An alpha particle spectrum (using a ${ }^{241} \mathrm{Am}$ source) was recorded at room temperature, using a prototype detector with planar gold electrodes. While the detector showed polarization effect, the spectrum recorded at room temperature provides incentive to work towards further progressing the development of $\mathrm{BiI}_{3}$ radiation detectors. Electron mobility was estimated to be $433 \pm 79 \mathrm{~cm}^{2} /$ Vs. Hole mobility could not be estimated due to lack of signal generated by holes. Charge carrier mobility depends on the concentration of traps and defects in the detector crystal. Thus it may be hypothesized that the increased mobility that was measured may be due to the increased purity of the crystal, which in turn can improve crystal quality thereby resulting in a detector crystal with fewer traps and defects.

Further improvement in material quality and detector fabrication techniques should improve the detector performance. Improvement in the electrical characteristics of the detectors may be achieved by reducing the internal defects (by introducing a doping agent, for example) and by subjecting the detector to surface treatment such as polishing and etching. Fabricating detectors with different electrode geometries to enable single polarity charge sensing techniques, would circumvent the problem of poor hole transport.

\section{Acknowledgements}

This project is supported by the Department of Energy National Nuclear Security Administration under contract DE-FG52-09NA29358.

\section{References}

[1] T. E. Schlesinger and R. B. James, Semiconductors for Room Temperature Nuclear Detector Applications, San Diego: Academic Press, 1995.

[2] D. S. McGregor and H. Hermon, Room-temperature compound semiconductor radiaition detectors, Nucl. Instr. Meth. A 395 (1997) 101. 
[3] T. Shoji, H. Onabe, and Y. Hiratate, Evaluation of CdTe(Cl) crystal growth with THM and application to a multichannel detector, Nucl. Instr. Meth. A 322 (1992) 324.

[4] K. Hitomi et al., Recent progress in thallium bromide detectors for $x$-ray and gamma-ray spectroscopy, Nucl. Instr. Meth. A 458 (2001) 365.

[5] A. T. Lintereur, W. Qiu, J. C. Nino, and J. E. Baciak, Iodine based compound semiconductors for room temperature gamma-ray spectroscopy, SPIE Proc. 6945 (2008) 694503-1.

[6] Y. N. Dmitriev, P. R. Bennett, L. J. Cirignano, M. Klugerman, and K. S. Shah, Bismuth Iodide crystals as a detector material: Some optical and electrical properties, SPIE Proc. 3768 (1999) 521.

[7] M. Matsumoto, K. Hitomi, T. Shoji, and Y. Hiratate, Bismuth tri-iodide crystal for nuclear radiation detectors, IEEE Trans. Nucl. Sci. 49 (2002) 2517.

[8] W. Qiu et al., Band gap and structure of single crystal BiI ${ }_{3}$ : Resolving discrepancies in literature, J. Appl. Phys. 114 (2013) 033110.

[9] L. Fornaro, I. Aguiar, A. Noguera, M. Perez and M. Rodriguez, Improvements on Bismuth TriIodide platelets for room temperature x-ray detection, IEEE NSS Conf. Rec. R04-3 (2006) 361.

[10] H. Yorikawa and S. Muramatsu, Theoretical study of crystal and electronic structures of BiI3, J. Phys.: Condens. Matter 20 (2008) 5220.

[11] M. Hostettler and D. Schwarzenbach, Phase diagrams and structures of $\mathrm{HgX} 2(X=I, B r, C l, F), C$. R. Chim 8 (2005) 147.

[12] A. V. Churilov et al., TlBr and $T l B r_{x} I_{1-x}$ crystals for $\gamma$-ray detectors, J. Cryst. Growth. 312 (2010) 1221.

[13] A. V. Churilov et al., Thallium bromide nuclear radiation detector development, IEEE Trans. Nucl. Sci. 56 (2009) 1875.

[14] A. Cuna et al., Purification of Bismuth Tri-Iodide as a materialfor radiation detector purposes, IEEE NSS Conf. Rec. 5 (2003) 3425.

[15] I. Beinglass, G. Dishon, A. Holzer, and M. Shieber, Improved crystals of mercuric iodide grown in a horizontal furnace from the vapor phase using the temperature oscillation method, J. Cryst. Growth, 42 (1977) 166.

[16] A. T. Lintereur, W. Qiu, J. C. Nino, and J. E. Baciak, Characterization of bismuth tri-iodide single crystals for wide band-gap semiconductor radiation detectors, Nucl. Instr. Meth. A 652 (2011) 166.

[17] W. Qiu, G. J. Dudder, X. Y. Zhao, S. S. Perry, and J. C. Nino, Interfacial reactivity of Au, Pd, and Pt on BiI(3) (001): implications for electrode selection, Acs. Appl. Mater. Inter. 3 (2011) 1910.

[18] L. Fornaro, A. Cuna, A. Noguera, M. Perez, and L. Mussio, Growth of Bismuth Tri-Iodide platelets for room temperature X-ray detection, IEEE Trans. Nucl. Sci. 51 (2004) 2461.

[19] L. Fornaro, E. Saucedo, L. Mussio, A. Gancharov, and A. Cuna, Bismuth triiodide polycrystalline films for digital X-ray radiography applications, IEEE Trans. Nucl. Sci. 51 (2004) 96.

[20] W. Qiu, Growth and characterization of bismuth tri-iodide single crystals by modified vertical Bridgman method, Ph.D. dissertation, Univ. Florida, 2010. 
[21] A. Cuna, N. Noguera, E. Saucedo, and L. Fornaro, Growth of bismuth tri-iodide platelets by the physical vapor deposition method, Cryst. Res. Technol. 39 (2004) 912.

[22] I. Aguiar, S. Kroger, and L. Fornaro, Bismuth tri-iodide polycrystalline films for $x$-ray direct and digital imagers, Nucl. Instr. Meth. A 610 (2009) 332.

[23] D. Nason and L. Keller, The growth and crystallography of bismuth tri-iodide crystals grown by vapor transport, J. Cryst. Growth. 156 (1995) 221.

[24] H. J. Kim, S. Khan, and H. Park, Zone refining and crystal growth of bismuth tri-iodide crystals, IEEE NSS Conf. Rec. R04-7 (2013) 1.

[25] Z. He, Review of the Schockley-Ramo theorem and its application in semiconductor gamma-ray detectors, Nucl. Instr. Meth. A 463 (2001) 250. 